\title{
Picone identities for half-linear elliptic operators with $p(x)$-Laplacians and applications to Sturmain comparison theory is
}

\author{
Norio Yoshida* \\ Department of Mathematics, University of Toyama, Toyama 930-8555, Japan
}

\begin{abstract}
Picone identities are established for a class of half-linear elliptic operators with $p(x)$-Laplacians, and Sturmian comparison theorems are obtained on the basis of the Picone identities. Generalizations to half-linear elliptic inequalities with mixed nonlinearities are discussed, and specializations to half-linear partial or ordinary differential inequalities with $p(x)$-Laplacians are shown.
\end{abstract}

Key words: $p(x)$-Laplacian, Picone identity, Picone-type inequality, half-linear, elliptic, Sturmian comparison theory

2000 MSC: 35B05, 35J92

\section{Introduction}

The operator $-\nabla \cdot\left(|\nabla u|^{p(x)-2} \nabla u\right)$ is said to be $p(x)$-Laplacian, and becomes $p$-Laplacian $-\nabla \cdot\left(|\nabla u|^{p-2} \nabla u\right)$ if $p(x)=p$ (constant), where the dot $\cdot$ denotes the scalar product, $\nabla=\left(\partial / \partial x_{1}, \ldots, \partial / \partial x_{n}\right)$ and $|x|$ denotes the Euclidean length of $x \in \mathbb{R}^{n}$. There has been much current interest in studying various mathematical problems with variable exponent growth condition. The study of such problems arise from nonlinear elasticity theory, electrorheological fluids (cf. [20, 27]).

Existence of weak solutions of the elliptic equation with $p(x)$-Laplacian

$$
-\nabla \cdot\left(a(x)|\nabla u|^{p(x)-2} \nabla u\right)+|u|^{p(x)-2} u=f(x, u) \text { in } \mathbb{R}^{n}
$$

were investigated by several authors, see, for example, $[5,7,14,25]$. For the existence of weak solutions for $p(x)$-Laplacian Dirichlet problem, we refer to $[8,13,15,16]$.

\footnotetext{
This research was partially supported by Grant-in-Aid for Scientific Research (C)(No. 20540159), the Ministry of Education, Culture, Sports, Science and Technology, Japan.

* Corresponding author

Email addresses: nori@sci.u-toyama.ac.jp (Norio Yoshida)
} 
The paper [26] by Zhang seems to be the first paper dealing with oscillations of solutions of $p(x)$-Laplacian equations. In [26] oscillation problem for the $p(t)$ Laplacian equation

$$
\left(\left|u^{\prime}\right|^{p(t)-2} u^{\prime}\right)^{\prime}+t^{-\theta(t)} g(t, u)=0, \quad t>0
$$

was treated. Motivated by Zhang [26], we establish Picone identities and Sturmian comparison theorems for half-linear elliptic inequalities.

Sturmain comparison theorems for half-linear elliptic equations

$$
\begin{aligned}
& \nabla \cdot\left(a(x)|\nabla u|^{\alpha-1} \nabla u\right)+c(x)|u|^{\alpha-1} u=0, \\
& \nabla \cdot\left(A(x)|\nabla v|^{\alpha-1} \nabla v\right)+C(x)|v|^{\alpha-1} v=0,
\end{aligned}
$$

where $\alpha>0$, were derived by utilizing a Picone identity, where we means by half-linear that a solution multiplied by any constant is also a solution. We refer the reader to Allegretto [1], Allegretto and Huang [3, 4], Bognár and Došlý [6], Došlý [9], Dunninger [12], Kusano, Jaroš and Yoshida [19], Yoshida $[21,22,23,24]$ for Picone identities and Sturmian comparison theorems, and to Došlý [10], Došlý and Řehák [11] for half-linear ordinary differential equations.

It might be natural to consider more genaral elliptic equations

$$
\begin{aligned}
& \nabla \cdot\left(a(x)|\nabla u|^{\alpha(x)-1} \nabla u\right)+c(x)|u|^{\alpha(x)-1} u=0, \\
& \nabla \cdot\left(A(x)|\nabla v|^{\alpha(x)-1} \nabla v\right)+C(x)|v|^{\alpha(x)-1} v=0,
\end{aligned}
$$

where $\alpha(x)>0$, but the above equations are not half-linear if $\alpha(x)$ is not a constant. In order to obtain some oscillation results such as Sturmian comparison theorems, etc., which are generalizations of those of linear differential equations, we first determine a class of half-linear elliptic equations with $p(x)$-Laplacians.

The objective of this paper is to establish Picone identities for half-linear elliptic inequalities

$$
\begin{aligned}
& u q[u] \geq 0 \\
& v Q[v] \leq 0
\end{aligned}
$$

where $q$ and $Q$ are defined by

$$
\begin{aligned}
& q[u] \quad:=\nabla \cdot\left(a(x)|\nabla u|^{\alpha(x)-1} \nabla u\right)-a(x)(\log |u|)|\nabla u|^{\alpha(x)-1} \nabla \alpha(x) \cdot \nabla u \\
& +|\nabla u|^{\alpha(x)-1} b(x) \cdot \nabla u+c(x)|u|^{\alpha(x)-1} u, \\
& Q[v]:=\quad \nabla \cdot\left(A(x)|\nabla v|^{\alpha(x)-1} \nabla v\right)-A(x)(\log |v|)|\nabla v|^{\alpha(x)-1} \nabla \alpha(x) \cdot \nabla v \\
& +|\nabla v|^{\alpha(x)-1} B(x) \cdot \nabla v+C(x)|v|^{\alpha(x)-1} v,
\end{aligned}
$$

and derive Sturmian comparison theorems for $q$ and $Q$ by using the Picone identities. In Section 2 we first show that (1.1) and (1.2) are half-linear in the sense that a constant multiple of a solution $u$ [resp. $v$ ] is also a solution of (1.1) [resp. (1.2)] (see Proposition 2.1), and then establish Picone identities for $q$ and $Q$. We mention, in particular, the paper [2] by Allegretto in which Picone 
Identity arguments are used, and the formulae that are closely related to Picone identities in Section 2 are established.

In Section 3 we derive Sturmian comparison theorems for $q$ and $Q$, and Section 4 is devoted to specializations to the case $\alpha(x)=\alpha>0$, and to halflinear ordinary differential equations with $p(t)$-Laplacians which seems to be unknown.

\section{Picone identities}

Let $G$ be a bounded domain in $\mathbb{R}^{n}$ with piecewise smooth boundary $\partial G$. It is assumed that $a(x), A(x) \in C(\bar{G} ;(0, \infty)), b(x), B(x) \in C\left(\bar{G} ; \mathbb{R}^{n}\right), c(x), C(x) \in$ $C(\bar{G} ; \mathbb{R})$, and that $\alpha(x) \in C^{1}(\bar{G} ;(0, \infty))$.

The domain $\mathcal{D}_{q}(G)$ of $q$ is defined to be the set of all functions $u$ of class $C^{1}(\bar{G} ; \mathbb{R})$ such that $a(x)|\nabla u|^{\alpha(x)-1} \nabla u \in C^{1}\left(G ; \mathbb{R}^{n}\right) \cap C\left(\bar{G} ; \mathbb{R}^{n}\right)$. The domain $\mathcal{D}_{Q}(G)$ of $Q$ is defined similarly.

We note in (1.1) that $\log |u|$ has singularities at zeros of $u(x)$, but $u \log |u|$ is continuous at every zero $x_{0}$ if we define $u \log |u|=0$ at $x=x_{0}$, in view of the fact that $\lim _{\varepsilon \rightarrow+0} \varepsilon \log \varepsilon=0$. We make the similar remarks in (1.2).

We consider the elliptic inequalities

$$
\begin{aligned}
& u q[u] \geq 0 \quad \text { in } G, \\
& v Q[v] \leq 0 \quad \text { in } G,
\end{aligned}
$$

where $q$ and $Q$ are defined by (1.3) and (1.4).

By a solution $u$ [resp. $v$ ] of (2.1) [resp. (2.2)] we mean a function $u \in \mathcal{D}_{q}(G)$ $\left[\right.$ resp. $\left.v \in \mathcal{D}_{Q}(G)\right]$ which satisfies $(2.1)[$ resp. $(2.2)]$ in $G$.

Proposition 2.1. Elliptic inequalities (2.1) and (2.2) are half-linear in the sense that if $u$ and $v$ are solutions of (2.1) and (2.2), then $k u$ and $k v$ are also solutions of (2.1) and (2.2) for any constant $k$, respectively.

ProOF. It suffices to show that (2.1) is half-linear. Let $u$ be any solution of $(2.1)$, and $k(\neq 0)$ be any constant. It is easy to see that

$$
\begin{aligned}
q[k u]= & \nabla \cdot\left(|k|^{\alpha(x)-1} k a(x)|\nabla u|^{\alpha(x)-1} \nabla u\right) \\
& -a(x)\left(|k|^{\alpha(x)-1} k\right)(\log (|k||u|))|\nabla u|^{\alpha(x)-1} \nabla \alpha(x) \cdot \nabla u \\
& +\left(|k|^{\alpha(x)-1} k\right)|\nabla u|^{\alpha(x)-1} b(x) \cdot \nabla u \\
& +\left(|k|^{\alpha(x)-1} k\right) c(x)|u|^{\alpha(x)-1} u .
\end{aligned}
$$

A simple computation shows that

$$
\begin{aligned}
& \nabla \cdot\left(|k|^{\alpha(x)-1} k a(x)|\nabla u|^{\alpha(x)-1} \nabla u\right) \\
&= \nabla\left(|k|^{\alpha(x)-1} k\right) \cdot\left(a(x)|\nabla u|^{\alpha(x)-1} \nabla u\right) \\
&+|k|^{\alpha(x)-1} k \nabla \cdot\left(a(x)|\nabla u|^{\alpha(x)-1} \nabla u\right) .
\end{aligned}
$$


Since

$$
\nabla\left(|k|^{\alpha(x)-1} k\right)=|k|^{\alpha(x)-1} k(\log |k|) \nabla \alpha(x),
$$

we see that

$$
\begin{aligned}
& \nabla \cdot\left(|k|^{\alpha(x)-1} k a(x)|\nabla u|^{\alpha(x)-1} \nabla u\right) \\
= & a(x)|k|^{\alpha(x)-1} k(\log |k|)|\nabla u|^{\alpha(x)-1} \nabla \alpha(x) \cdot \nabla u \\
& +|k|^{\alpha(x)-1} k \nabla \cdot\left(a(x)|\nabla u|^{\alpha(x)-1} \nabla u\right) .
\end{aligned}
$$

Combining (2.3) and (2.5) yields

$$
(k u) q[k u]=|k|^{\alpha(x)+1} u q[u] \geq 0
$$

for any constant $k(\neq 0)$. Since $(k u) \log |k u|=0$ for $k=0$, we easily see that $(k u) q[k u]=0$ for $k=0$. Hence, we conclude that $(2.1)$ is half-linear.

Remark 2.1. We note that (2.1) and (2.2) are half-linear if and only if $u q[u]$ and $v Q[v]$ are "homogeneous" functions in $u$ and $v$, respectively, which satisfy

$$
\begin{aligned}
& (k u) q[k u]=|k|^{\alpha(x)+1} u q[u](k \in \mathbb{R}), \\
& (k v) Q[k v]=|k|^{\alpha(x)+1} v Q[v](k \in \mathbb{R}) .
\end{aligned}
$$

Theorem 2.1 (Picone identity for $Q$ ). If $v \in \mathcal{D}_{Q}(G)$ and $v$ has no zero in $G$, then we obtain the following Picone identity for any $u \in C^{1}(G ; \mathbb{R})$ :

$$
\begin{gathered}
-\nabla \cdot\left(u \varphi(u) \frac{A(x)|\nabla v|^{\alpha(x)-1} \nabla v}{\varphi(v)}\right) \\
=-A(x)\left|\nabla u+\frac{u \log |u|}{\alpha(x)+1} \nabla \alpha(x)-\frac{u}{(\alpha(x)+1) A(x)} B(x)\right|^{\alpha(x)+1} \\
+C(x)|u|^{\alpha(x)+1} \\
+A(x)|| \nabla u+\frac{u \log |u|}{\alpha(x)+1} \nabla \alpha(x)-\left.\frac{u}{(\alpha(x)+1) A(x)} B(x)\right|^{\alpha(x)+1} \\
\quad+\alpha(x)\left|\frac{u}{v} \nabla v\right|^{\alpha(x)+1} \\
\quad-(\alpha(x)+1)\left|\frac{u}{v} \nabla v\right|^{\alpha(x)-1}\left(\nabla u+\frac{u \log |u|}{\alpha(x)+1} \nabla \alpha(x)\right. \\
-\frac{|u|^{\alpha(x)+1}}{|v|^{\alpha(x)+1}}(v Q[v]) \quad \text { in } G, \\
\text { where } \varphi(u)=|u|^{\alpha(x)-1} u=|u(x)|^{\alpha(x)-1} u(x) .
\end{gathered}
$$


Proof. A direct calculation yields

$$
\begin{aligned}
& -\nabla \cdot\left(u \varphi(u) \frac{A(x)|\nabla v|^{\alpha(x)-1} \nabla v}{\varphi(v)}\right) \\
= & -\nabla(u \varphi(u)) \cdot \frac{A(x)|\nabla v|^{\alpha(x)-1} \nabla v}{\varphi(v)} \\
& -u \varphi(u) A(x)|\nabla v|^{\alpha(x)-1} \nabla\left(\frac{1}{\varphi(v)}\right) \cdot \nabla v \\
& -\frac{u \varphi(u)}{\varphi(v)} \nabla \cdot\left(A(x)|\nabla v|^{\alpha(x)-1} \nabla v\right) .
\end{aligned}
$$

We easily see that

$$
\begin{aligned}
& \nabla(u \varphi(u))=(\alpha(x)+1) \varphi(u) \nabla u+u \varphi(u)(\log |u|) \nabla \alpha(x), \\
& \nabla\left(\frac{1}{\varphi(v)}\right)=-\frac{\alpha(x)}{v \varphi(v)} \nabla v-\frac{\log |v|}{\varphi(v)} \nabla \alpha(x)
\end{aligned}
$$

in view of the fact that

$$
\nabla \varphi(v)=\alpha(x) \frac{\varphi(v)}{v} \nabla v+(\log |v|) \varphi(v) \nabla \alpha(x) .
$$

Hence, we observe from (2.8) and (2.9) that

$$
\begin{aligned}
& \nabla(u \varphi(u)) \cdot \frac{A(x)|\nabla v|^{\alpha(x)-1} \nabla v}{\varphi(v)} \\
= & (\alpha(x)+1) \frac{\varphi(u)}{\varphi(v)} A(x)|\nabla v|^{\alpha(x)-1} \nabla u \cdot \nabla v \\
& +u \varphi(u)(\log |u|) \frac{A(x)|\nabla v|^{\alpha(x)-1}}{\varphi(v)} \nabla \alpha(x) \cdot \nabla v \\
= & (\alpha(x)+1) A(x)\left|\frac{u}{v} \nabla v\right|^{\alpha(x)-1}(\nabla u) \cdot\left(\frac{u}{v} \nabla v\right) \\
& +A(x) u(\log |u|) \frac{\varphi(u)}{\varphi(v)}|\nabla v|^{\alpha(x)-1} \nabla \alpha(x) \cdot \nabla v
\end{aligned}
$$

and

$$
\begin{aligned}
& u \varphi(u) A(x)|\nabla v|^{\alpha(x)-1} \nabla\left(\frac{1}{\varphi(v)}\right) \cdot \nabla v \\
= & -\alpha(x) \frac{u \varphi(u)}{v \varphi(v)} A(x)|\nabla v|^{\alpha(x)+1} \\
& -\frac{u \varphi(u)}{\varphi(v)} A(x)(\log |v|)|\nabla v|^{\alpha(x)-1} \nabla \alpha(x) \cdot \nabla v \\
= & -A(x) \alpha(x)\left|\frac{u}{v} \nabla v\right|^{\alpha(x)+1} \\
& -\frac{u \varphi(u)}{\varphi(v)} A(x)(\log |v|)|\nabla v|^{\alpha(x)-1} \nabla \alpha(x) \cdot \nabla v .
\end{aligned}
$$


It follows from (1.2) that

$$
\begin{aligned}
& \frac{u \varphi(u)}{\varphi(v)} \nabla \cdot\left(A(x)|\nabla v|^{\alpha(x)-1} \nabla v\right) \\
= & \frac{u \varphi(u)}{\varphi(v)}\left(Q[v]+A(x)(\log |v|)|\nabla v|^{\alpha(x)-1} \nabla \alpha(x) \cdot \nabla v\right. \\
& \left.-|\nabla v|^{\alpha(x)-1} B(x) \cdot \nabla v-C(x)|v|^{\alpha(x)-1} v\right) \\
= & \frac{u \varphi(u)}{\varphi(v)} Q[v]+\frac{u \varphi(u)}{\varphi(v)} A(x)(\log |v|)|\nabla v|^{\alpha(x)-1} \nabla \alpha(x) \cdot \nabla v \\
& -\frac{u \varphi(u)}{\varphi(v)}|\nabla v|^{\alpha(x)-1} B(x) \cdot \nabla v-C(x)|u|^{\alpha(x)+1} .
\end{aligned}
$$

Combining (2.7), (2.10)-(2.12), we arrive at

$$
\begin{aligned}
& -\nabla \cdot\left(u \varphi(u) \frac{A(x)|\nabla v|^{\alpha(x)-1} \nabla v}{\varphi(v)}\right) \\
= & C(x)|u|^{\alpha(x)+1} \\
& +A(x)\left[\alpha(x)\left|\frac{u}{v} \nabla v\right|^{\alpha(x)+1}-(\alpha(x)+1)\left|\frac{u}{v} \nabla v\right|^{\alpha(x)-1}(\nabla u) \cdot\left(\frac{u}{v} \nabla v\right)\right] \\
& -A(x) u(\log |u|)\left|\frac{u}{v} \nabla v\right|^{\alpha(x)-1}(\nabla \alpha(x)) \cdot\left(\frac{u}{v} \nabla v\right) \\
& +u\left|\frac{u}{v} \nabla v\right|^{\alpha(x)-1} B(x) \cdot\left(\frac{u}{v} \nabla v\right) \\
& -\frac{u}{\varphi(v)}(\varphi(u) Q[v]) \\
= & C(x)|u|^{\alpha(x)+1} \\
& \left.+A(x)\left[\alpha(x)\left|\frac{u}{v} \nabla v\right|^{\alpha(x)+1} \quad-\frac{u}{(\alpha(x)+1) A(x)} B(x)\right) \cdot\left(\frac{u}{v} \nabla v\right)\right] \\
& -\frac{u \varphi(u)}{v \varphi(v)}(v Q[v]),
\end{aligned}
$$

which is equivalent to the desired identity (2.6).

Now we consider the first-order differential system

$$
\nabla w=H(x),
$$

where $H(x)=\left(h_{1}(x), h_{2}(x), \ldots, h_{n}(x)\right)$ is a vector function of class $C^{1}$, and we define the sequence of functions $\left\{g_{k}(x)\right\}_{k=1}^{n}$ by

$$
g_{1}(x)=\int h_{1}(x) d x_{1},
$$




$$
g_{k}(x)=g_{k-1}(x)+\int\left(h_{k}(x)-\frac{\partial}{\partial x_{k}} g_{k-1}(x)\right) d x_{k} \quad(k=2,3, \ldots, n) .
$$

Proposition 2.2. The system (2.13) has a $C^{1}$-solution if and only if

$$
\frac{\partial}{\partial x_{j-1}}\left(h_{k}(x)-\frac{\partial}{\partial x_{k}} g_{k-1}(x)\right)=0 \quad(j=2,3, \ldots, k ; k=2,3, \ldots, n) .
$$

Then any $C^{1}$-solution $w$ of (2.13) has the form

$$
w=g_{n}(x)+C_{n}
$$

for some constant $C_{n}$.

Proof. Assume that (2.13) has a $C^{1}$-solution $w$, then we obtain

$$
\frac{\partial w}{\partial x_{1}}=h_{1}(x)
$$

and

$$
\begin{aligned}
w & =\int h_{1}(x) d x_{1}+C_{1}\left(x_{2}, \ldots, x_{n}\right) \\
& =g_{1}(x)+C_{1}\left(x_{2}, \ldots, x_{n}\right)
\end{aligned}
$$

for some function $C_{1}\left(x_{2}, \ldots, x_{n}\right)$. Since we have

$$
\frac{\partial w}{\partial x_{2}}=h_{2}(x)
$$

we find that $C_{1}\left(x_{2}, \ldots, x_{n}\right)$ must satisfy

$$
\frac{\partial C_{1}}{\partial x_{2}}=h_{2}(x)-\frac{\partial}{\partial x_{2}} g_{1}(x) .
$$

It is necessary that

$$
\frac{\partial}{\partial x_{1}}\left(h_{2}(x)-\frac{\partial}{\partial x_{2}} g_{1}(x)\right)=0
$$

and we obtain

$$
C_{1}=\int\left(h_{2}(x)-\frac{\partial}{\partial x_{2}} g_{1}(x)\right) d x_{2}+C_{2}\left(x_{3}, \ldots, x_{n}\right)
$$

for some function $C_{2}\left(x_{3}, \ldots, x_{n}\right)$, and hence

$$
\begin{aligned}
w & =g_{1}(x)+\int\left(h_{2}(x)-\frac{\partial}{\partial x_{2}} g_{1}(x)\right) d x_{2}+C_{2}\left(x_{3}, \ldots, x_{n}\right) \\
& =g_{2}(x)+C_{2}\left(x_{3}, \ldots, x_{n}\right) .
\end{aligned}
$$

Repeating the above procedure, we observe that (2.15) is necessary that the solution $w$ can be written in the form (2.17). It can be shown from the above consideration that the condition (2.16) is sufficient for (2.13) to have a $C^{1}$ solution. 
Theorem 2.2 (Picone identity for $q$ and $Q)$. Let $\alpha(x) \in C^{2}(G ;(0, \infty))$ and $b(x) / a(x) \in C^{1}\left(G ; \mathbb{R}^{n}\right)$. Assume that $u \in C^{1}(G ; \mathbb{R})$, $u$ has no zero in $G$, and that:

$\left(\mathrm{H}_{1}\right)$ there is a function $f \in C(\bar{G} ; \mathbb{R})$ such that $f \in C^{1}(G ; \mathbb{R})$ and

$$
\nabla f=\frac{\log |u|}{\alpha(x)+1} \nabla \alpha(x)-\frac{b(x)}{(\alpha(x)+1) a(x)} \quad \text { in } G .
$$

If $e^{f} u \in \mathcal{D}_{q}(G), v \in \mathcal{D}_{Q}(G)$ and $v$ has no zero in $G$, then we obtain the following Picone identity:

$$
\begin{aligned}
& \nabla \cdot\left(e^{-(\alpha(x)+1) f}\left(e^{f} u\right) a(x)\left|\nabla\left(e^{f} u\right)\right|^{\alpha(x)-1} \nabla\left(e^{f} u\right)\right. \\
& \left.-\frac{u \varphi(u)}{\varphi(v)} A(x)|\nabla v|^{\alpha(x)-1} \nabla v\right) \\
& =a(x)\left|\nabla u+\frac{u \log |u|}{\alpha(x)+1} \nabla \alpha(x)-\frac{u}{(\alpha(x)+1) a(x)} b(x)\right|^{\alpha(x)+1} \\
& -A(x)\left|\nabla u+\frac{u \log |u|}{\alpha(x)+1} \nabla \alpha(x)-\frac{u}{(\alpha(x)+1) A(x)} B(x)\right|^{\alpha(x)+1} \\
& +(C(x)-c(x))|u|^{\alpha(x)+1} \\
& +A(x)\left[\left|\nabla u+\frac{u \log |u|}{\alpha(x)+1} \nabla \alpha(x)-\frac{u}{(\alpha(x)+1) A(x)} B(x)\right|^{\alpha(x)+1}\right. \\
& +\alpha(x)\left|\frac{u}{v} \nabla v\right|^{\alpha(x)+1} \\
& -(\alpha(x)+1)\left|\frac{u}{v} \nabla v\right|^{\alpha(x)-1}\left(\nabla u+\frac{u \log |u|}{\alpha(x)+1} \nabla \alpha(x)\right. \\
& \left.\left.-\frac{u}{(\alpha(x)+1) A(x)} B(x)\right) \cdot\left(\frac{u}{v} \nabla v\right)\right] \\
& +e^{-(\alpha(x)+1) f}\left(e^{f} u\right) q\left[e^{f} u\right]-\frac{|u|^{\alpha(x)+1}}{|v|^{\alpha(x)+1}}(v Q[v]) \quad \text { in } G .
\end{aligned}
$$

Proof. A direct calculation shows that

$$
\begin{aligned}
& \nabla \cdot\left(e^{-(\alpha(x)+1) f}\left(e^{f} u\right) a(x)\left|\nabla\left(e^{f} u\right)\right|^{\alpha(x)-1} \nabla\left(e^{f} u\right)\right) \\
= & \left(e^{f} u\right) \nabla\left(e^{-(\alpha(x)+1) f}\right) \cdot\left(a(x)\left|\nabla\left(e^{f} u\right)\right|^{\alpha(x)-1} \nabla\left(e^{f} u\right)\right) \\
& +e^{-(\alpha(x)+1) f} \nabla\left(e^{f} u\right) \cdot\left(a(x)\left|\nabla\left(e^{f} u\right)\right|^{\alpha(x)-1} \nabla\left(e^{f} u\right)\right) \\
& +e^{-(\alpha(x)+1) f}\left(e^{f} u\right) \nabla \cdot\left(a(x)\left|\nabla\left(e^{f} u\right)\right|^{\alpha(x)-1} \nabla\left(e^{f} u\right)\right) .
\end{aligned}
$$

Since

$$
\nabla\left(e^{-(\alpha(x)+1) f}\right)=e^{-(\alpha(x)+1) f}(-(\nabla \alpha(x)) f-(\alpha(x)+1) \nabla f),
$$


we observe, using the hypothesis $\left(\mathrm{H}_{1}\right)$, that

$$
\begin{aligned}
& \left(e^{f} u\right) \nabla\left(e^{-(\alpha(x)+1) f}\right) \\
= & e^{-(\alpha(x)+1) f}\left[-\left(e^{f} u\right)(\nabla \alpha(x)) f-(\alpha(x)+1) e^{f} u \nabla f\right] \\
= & e^{-(\alpha(x)+1) f}\left[-\left(e^{f} u\right)(\nabla \alpha(x)) f-e^{f}(u \log |u|) \nabla \alpha(x)+e^{f} \frac{u}{a(x)} b(x)\right] \\
= & e^{-(\alpha(x)+1) f}\left(e^{f} u\right)\left[-\log \left|e^{f} u\right| \nabla \alpha(x)+\frac{b(x)}{a(x)}\right]
\end{aligned}
$$

and therefore

$$
\begin{aligned}
&\left(e^{f} u\right) \nabla\left(e^{-(\alpha(x)+1) f}\right) \cdot\left(a(x)\left|\nabla\left(e^{f} u\right)\right|^{\alpha(x)-1} \nabla\left(e^{f} u\right)\right) \\
&=e^{-(\alpha(x)+1) f}\left(e^{f} u\right)[-a(x) \log \left|e^{f} u\right|\left|\nabla\left(e^{f} u\right)\right|^{\alpha(x)-1} \nabla \alpha(x) \cdot \nabla\left(e^{f} u\right) \\
&\left.+\left|\nabla\left(e^{f} u\right)\right|^{\alpha(x)-1} b(x) \cdot \nabla\left(e^{f} u\right)\right] .
\end{aligned}
$$

It is clear that

$$
\begin{aligned}
& e^{-(\alpha(x)+1) f} \nabla\left(e^{f} u\right) \cdot a(x)\left|\nabla\left(e^{f} u\right)\right|^{\alpha(x)-1} \nabla\left(e^{f} u\right) \\
= & e^{-(\alpha(x)+1) f} a(x)\left|\nabla\left(e^{f} u\right)\right|^{\alpha(x)+1} \\
= & a(x)\left|e^{-f} \nabla\left(e^{f} u\right)\right|^{\alpha(x)+1} \\
= & a(x)|\nabla u+u \nabla f|^{\alpha(x)+1} \\
= & a(x)\left|\nabla u+\frac{u \log |u|}{\alpha(x)+1} \nabla \alpha(x)-\frac{u}{(\alpha(x)+1) a(x)} b(x)\right|^{\alpha(x)+1}
\end{aligned}
$$

in view of the hypothesis $\left(\mathrm{H}_{1}\right)$. From (2.19)-(2.21) it follows that

$$
\begin{aligned}
& \nabla \cdot\left(e^{-(\alpha(x)+1) f}\left(e^{f} u\right) a(x)\left|\nabla\left(e^{f} u\right)\right|^{\alpha(x)-1} \nabla\left(e^{f} u\right)\right) \\
& =e^{-(\alpha(x)+1) f}\left(e^{f} u\right)\left[\nabla \cdot\left(a(x)\left|\nabla\left(e^{f} u\right)\right|^{\alpha(x)-1} \nabla\left(e^{f} u\right)\right)\right. \\
& -a(x) \log \left|e^{f} u \| \nabla\left(e^{f} u\right)\right|^{\alpha(x)-1} \nabla \alpha(x) \cdot \nabla\left(e^{f} u\right) \\
& \left.+\left|\nabla\left(e^{f} u\right)\right|^{\alpha(x)-1} b(x) \cdot \nabla\left(e^{f} u\right)\right] \\
& +a(x)\left|\nabla u+\frac{u \log |u|}{\alpha(x)+1} \nabla \alpha(x)-\frac{u}{(\alpha(x)+1) a(x)} b(x)\right|^{\alpha(x)+1} \\
& =a(x)\left|\nabla u+\frac{u \log |u|}{\alpha(x)+1} \nabla \alpha(x)-\frac{u}{(\alpha(x)+1) a(x)} b(x)\right|^{\alpha(x)+1} \\
& +e^{-(\alpha(x)+1) f}\left(e^{f} u\right)\left[q\left[e^{f} u\right]-c(x)\left|e^{f} u\right|^{\alpha(x)-1} e^{f} u\right] \\
& =a(x)\left|\nabla u+\frac{u \log |u|}{\alpha(x)+1} \nabla \alpha(x)-\frac{u}{(\alpha(x)+1) a(x)} b(x)\right|^{\alpha(x)+1} \\
& -c(x)|u|^{\alpha(x)+1}+e^{-(\alpha(x)+1) f}\left(e^{f} u\right) q\left[e^{f} u\right] .
\end{aligned}
$$

Combining (2.6) with (2.22) yields the desired identity (2.18). 
Remark 2.2. In order to explain the role of the function $f$ in the hypothesis $\left(\mathrm{H}_{1}\right)$, we treat the ordinary differential operaor $\ell$ and the variation $V[y]$ defined by

$$
\begin{aligned}
& \ell[y]=\left(a(t) y^{\prime}\right)^{\prime}+b(t) y^{\prime}, \quad t \in\left(t_{1}, t_{2}\right), \\
& V[y]=\int_{t_{1}}^{t_{2}} a(t)\left|y^{\prime}-\frac{b(t)}{2 a(t)} y\right|^{2} d t,
\end{aligned}
$$

where $a(t) \in C^{1}\left(\left[t_{1}, t_{2}\right] ;(0, \infty)\right)$ and $b(t) \in C\left(\left[t_{1}, t_{2}\right] ; \mathbb{R}\right)$. Letting

$$
f(t)=-\int \frac{b(t)}{2 a(t)} d t
$$

we observe that

$$
\begin{aligned}
V[y] & =\int_{t_{1}}^{t_{2}} a(t)\left|e^{-f(t)}\left(e^{f(t)} y\right)^{\prime}\right|^{2} d t \\
& =\int_{t_{1}}^{t_{2}} e^{-2 f(t)} a(t)\left(\left(e^{f(t)} y\right)^{\prime}\right)^{2} d t \\
& =-\int_{t_{1}}^{t_{2}} e^{-2 f(t)}\left(e^{f(t)} y\right) \ell\left[e^{f(t)} y\right] d t
\end{aligned}
$$

if $y\left(t_{1}\right)=y\left(t_{2}\right)=0$. Introducing the function $f(t)$, we can consider the function $e^{f(t)} y$ to be a new unknown function.

Remark 2.3. We give an example which illustrates the hypothesis $\left(\mathrm{H}_{1}\right)$. Let $n=1, G=(0, \pi), u=\sin x, \alpha(x)=e^{\sin x+1}-1, a(x)=1, b(x)=-(\cos x) e^{\sin x+1}$. Defining $f(x)$ by

$$
f(x)=\left\{\begin{array}{cl}
(\sin x) \log \sin x, & x \in(0, \pi) \\
0 & \text { at } x=0, \pi,
\end{array}\right.
$$

we conclude that

$$
\begin{aligned}
f^{\prime}(x) & =(\cos x) \log \sin x+\cos x \\
& =\frac{\log |u|}{\alpha(x)+1} \alpha^{\prime}(x)-\frac{b(x)}{(\alpha(x)+1) a(x)} \quad \text { in }(0, \pi) .
\end{aligned}
$$

Moreover, we see that $f(x)$ is a continuous function on $[0, \pi]$ in view of the fact that $\lim _{\varepsilon \rightarrow+0} \varepsilon \log \varepsilon=0$.

Remark 2.4. It follows from Proposition 2.2 that if $\left(\mathrm{H}_{1}\right)$ holds, then the function

$$
\frac{\log |u|}{\alpha(x)+1} \nabla \alpha(x)-\frac{b(x)}{(\alpha(x)+1) a(x)}
$$

must satisfy (2.16) in $G$ with $H(x)$ replaced by (2.23). It is necessary that $\alpha(x) \in C^{2}$ and $b(x) / a(x) \in C^{1}$. For example, we treat the case where $n=2$, $G=(0, \pi) \times(0, \pi), u=\sin x_{1} \sin x_{2}, \alpha(x)=e^{\sin x_{1} \sin x_{2}+1}-1, a(x)=1$, and

$$
b(x)=\left(-\left(\cos x_{1} \sin x_{2}\right) e^{\sin x_{1} \sin x_{2}+1},-\left(\sin x_{1} \cos x_{2}\right) e^{\sin x_{1} \sin x_{2}+1}\right) .
$$


Then we have

$$
\frac{\log |u|}{\alpha(x)+1} \nabla \alpha(x)-\frac{b(x)}{(\alpha(x)+1) a(x)}=\left(h_{1}\left(x_{1}, x_{2}\right), h_{2}\left(x_{1}, x_{2}\right)\right),
$$

where

$$
\begin{aligned}
& h_{1}\left(x_{1}, x_{2}\right)=\left(\cos x_{1} \log \sin x_{1}+\cos x_{1}\right) \sin x_{2}+\cos x_{1} \sin x_{2} \log \sin x_{2}, \\
& h_{2}\left(x_{1}, x_{2}\right)=\left(\cos x_{2} \log \sin x_{2}+\cos x_{2}\right) \sin x_{1}+\cos x_{2} \sin x_{1} \log \sin x_{1} .
\end{aligned}
$$

It is easy to check that

$$
\frac{\partial}{\partial x_{1}}\left(h_{2}\left(x_{1}, x_{2}\right)-\frac{\partial}{\partial x_{2}} \int h_{1}\left(x_{1}, x_{2}\right) d x_{1}\right)=0,
$$

and the solution $f$ of

$$
\nabla f=\left(h_{1}\left(x_{1}, x_{2}\right), h_{2}\left(x_{1}, x_{2}\right)\right) \quad \text { in } G
$$

is written in the form

$$
f=\left(\sin x_{1} \log \sin x_{1}\right) \sin x_{2}+\left(\sin x_{2} \log \sin x_{2}\right) \sin x_{1},
$$

which is continuous on $\bar{G}=[0, \pi] \times[0, \pi]$ by defining $f=0$ on $\partial G$.

\section{Sturmian comparison theorems}

On the basis of the Picone identity in Section 2 we present Sturmian comparison theorems for the half-linear elliptic operators $q$ and $Q$.

Lemma 3.1. The inequality

$$
|\xi|^{\alpha(x)+1}+\alpha(x)|\eta|^{\alpha(x)+1}-(\alpha(x)+1)|\eta|^{\alpha(x)-1} \xi \cdot \eta \geq 0
$$

is valid for $x \in G, \xi, \eta \in \mathbb{R}^{n}$, where the equality holds if and only if $\xi=\eta$.

Proof. For any fixed $x \in G$, the inequality (3.1) holds for any $\xi, \eta \in \mathbb{R}^{n}$ by Hardy, Littlewood and Pólya [17, Theorem 41] and Kusano, Jaroš and Yoshida [19, Lemma 2.1].

Theorem 3.1 (Sturmian comparison theorem). Let $\alpha(x) \in C^{2}(G ;(0, \infty))$ and $b(x) / a(x), B(x) / A(x) \in C^{1}\left(G ; \mathbb{R}^{n}\right)$. Assume that there exists a function $u \in C^{1}(\bar{G} ; \mathbb{R})$ such that $u=0$ on $\partial G, u$ has no zero in $G$, the hypothesis $\left(\mathrm{H}_{1}\right)$ of Theorem 2.2 holds and that:

$\left(\mathrm{H}_{2}\right)$ there is a function $F \in C(\bar{G} ; \mathbb{R})$ such that $F \in C^{1}(G ; \mathbb{R})$ and

$$
\nabla F=\frac{\log |u|}{\alpha(x)+1} \nabla \alpha(x)-\frac{B(x)}{(\alpha(x)+1) A(x)} \quad \text { in } G .
$$


If the following conditions are satisfied:

(i) $e^{f} u \in \mathcal{D}_{q}(G)$ and

$$
\left(e^{f} u\right) q\left[e^{f} u\right] \geq 0 \quad \text { in } G
$$

(ii)

$$
\begin{aligned}
V_{G}[u]:=\int_{G}[ & a(x)\left|\nabla u+\frac{u \log |u|}{\alpha(x)+1} \nabla \alpha(x)-\frac{u}{(\alpha(x)+1) a(x)} b(x)\right|^{\alpha(x)+1} \\
& -A(x)\left|\nabla u+\frac{u \log |u|}{\alpha(x)+1} \nabla \alpha(x)-\frac{u}{(\alpha(x)+1) A(x)} B(x)\right|^{\alpha(x)+1} \\
& \left.+(C(x)-c(x))|u|^{\alpha(x)+1}\right] d x \geq 0,
\end{aligned}
$$

then every solution $v \in \mathcal{D}_{Q}(G)$ of (2.2) must vanish at some point of $\bar{G}$.

Proof. Suppose to the contrary that there exists a solution $v \in \mathcal{D}_{Q}(G)$ of $(2.2)$ such that $v$ has no zero on $\bar{G}$. Integrating the Picone identity (2.18) over $G$ and using the divergence theorem, we obtain

$$
0 \geq V_{G}[u]+\int_{G} W(u, v) d x \geq 0,
$$

which yields the following

$$
\int_{G} W(u, v) d x=0
$$

where

$$
\begin{aligned}
W(u, v) & \\
:=\quad A(x)[\mid \nabla & u+\frac{u \log |u|}{\alpha(x)+1} \nabla \alpha(x)-\left.\frac{u}{(\alpha(x)+1) A(x)} B(x)\right|^{\alpha(x)+1} \\
& +\alpha(x)\left|\frac{u}{v} \nabla v\right|^{\alpha(x)+1} \\
& -(\alpha(x)+1)\left|\frac{u}{v} \nabla v\right|^{\alpha(x)-1}\left(\nabla u+\frac{u \log |u|}{\alpha(x)+1} \nabla \alpha(x)\right. \\
& \left.\left.-\frac{u}{(\alpha(x)+1) A(x)} B(x)\right) \cdot\left(\frac{u}{v} \nabla v\right)\right] .
\end{aligned}
$$

It follows from Lemma 3.1 that

$$
\nabla u+\frac{u \log |u|}{\alpha(x)+1} \nabla \alpha(x)-\frac{u}{(\alpha(x)+1) A(x)} B(x) \equiv \frac{u}{v} \nabla v \quad \text { in } G,
$$

that is,

$$
\nabla u+u \nabla F \equiv \frac{u}{v} \nabla v \quad \text { in } G
$$


from which we have

$$
e^{-F} v \nabla\left(e^{F} \frac{u}{v}\right) \equiv 0 \quad \text { in } G .
$$

Therefore, there exists a constant $k_{0}$ such that $e^{F} u / v=k_{0}$ in $G$ and hence on $\bar{G}$ by continuity. Since $u=0$ on $\partial G$, we see that $k_{0}=0$, which contradicts the hypothesis that $u$ is nontrivial. The proof is complete.

Corollary 3.1. Let $\alpha(x) \in C^{2}(G ;(0, \infty)), b(x) / a(x), B(x) / A(x) \in C^{1}\left(G ; \mathbb{R}^{n}\right)$. Assume that:

(i) $\frac{b(x)}{a(x)}=\frac{B(x)}{A(x)}$ in $G$;

(ii) $a(x) \geq A(x), C(x) \geq c(x) \quad$ in $G$.

If there exists a function $u \in C^{1}(\bar{G} ; \mathbb{R})$ such that $u=0$ on $\partial G$, u has no zero in $G$, the hypothesis $\left(\mathrm{H}_{1}\right)$ of Theorem 2.2 holds and (i) of Theorem 3.1 is satisfied, then every solution $v \in \mathcal{D}_{Q}(G)$ of (2.2) must vanish at some point of $\bar{G}$.

Proof. The conditions (i), (ii) imply that $V_{G}[u] \geq 0$ for any $u \in C^{1}(\bar{G} ; \mathbb{R})$ and $\left(\mathrm{H}_{2}\right)$ is the same as $\left(\mathrm{H}_{1}\right)$. The conclusion follows from Theorem 3.1.

Corollary 3.2. Let $\alpha(x) \in C^{2}(G ;(0, \infty)), b(x) / a(x), B(x) / A(x) \in C^{1}\left(G ; \mathbb{R}^{n}\right)$. Assume that the hypotheses (i), (ii) of Corollary 3.1 are satisfied, and that there exists a nontrivial function $u \in C^{1}(\bar{G} ; \mathbb{R})$ which satisfies $u=0$ on $\partial G$ and the following:

$\left(\tilde{\mathrm{H}}_{1}\right)$ there is a function $f \in C(\bar{G} ; \mathbb{R})$ such that $f \in C^{1}\left(N_{u} ; \mathbb{R}\right)$ and

$$
\nabla f=\frac{\log |u|}{\alpha(x)+1} \nabla \alpha(x)-\frac{b(x)}{(\alpha(x)+1) a(x)} \quad \text { in } N_{u},
$$

where

$$
N_{u}:=\{x \in G ; u(x) \neq 0\} .
$$

If $e^{f} u \in \mathcal{D}_{q}\left(N_{u}\right),\left(e^{f} u\right) q\left[e^{f} u\right] \geq 0$ in $N_{u}$, then every solution $v \in \mathcal{D}_{Q}(G)$ of (2.2) must vanish at some point of $\bar{G}$.

Proof. Since $u$ is nontrivial and $u=0$ on $\partial G$, there is a domain $G_{0} \subset G$ for which $u=0$ on $\partial G_{0}$ and $u$ has no zero in $G_{0}$. Applying Corollary 3.1 with $G$ replaced by $G_{0}$, we conclude that every solution $v \in \mathcal{D}_{Q}(G)$ of $(2.2)$ must vanish at some point of $\overline{G_{0}} \subset \bar{G}$, that is, $v$ has a zero on $\bar{G}$. by

Next we deal with the case where $G$ is the annular domain $A\left(r_{1}, r_{2}\right)$ defined

$$
A\left(r_{1}, r_{2}\right)=\left\{x \in \mathbb{R}^{n} ; r_{1}<|x|<r_{2}\right\}\left(r_{1}<r_{2}\right) .
$$

We use the notation:

$$
\begin{aligned}
& A\left[r_{1}, r_{2}\right]=\left\{x \in \mathbb{R}^{n} ; r_{1} \leq|x| \leq r_{2}\right\}, \\
& S_{r}=\left\{x \in \mathbb{R}^{n} ;|x|=r\right\} .
\end{aligned}
$$


Let $\bar{A}(r)$ and $\bar{C}(r)$ denote the spherical means of $A(x)$ and $C(x)$ over the sphere $S_{r}$, respectively, that is,

$$
\begin{aligned}
& \bar{A}(r)=\frac{1}{\omega_{n} r^{n-1}} \int_{S_{r}} A(x) d S=\frac{1}{\omega_{n}} \int_{S_{1}} A(r, \theta) d \omega, \\
& \bar{C}(r)=\frac{1}{\omega_{n} r^{n-1}} \int_{S_{r}} C(x) d S=\frac{1}{\omega_{n}} \int_{S_{1}} C(r, \theta) d \omega,
\end{aligned}
$$

where $\omega_{n}$ is the surface area of the unit sphere $S_{1},(r, \theta)$ is the hyperspherical coordinates in $\mathbb{R}^{n}$ and $\omega$ is the measure on $S_{1}$.

We assume that:

$\left(\mathrm{H}_{3}\right) \alpha(x) \equiv \alpha(|x|) \quad$ in $A\left(r_{1}, r_{2}\right)$;

$\left(\mathrm{H}_{4}\right) \frac{B(x)}{A(x)}=B_{0}(|x|) \frac{x_{i}}{|x|} \quad$ in $A\left(r_{1}, r_{2}\right)$ for some function $B_{0}(r) \in C\left[r_{1}, r_{2}\right]$.

Associated with (2.2) we treat the half-linear elliptic operator $\tilde{q}$ defined by

$$
\begin{aligned}
\tilde{q}[u]:=\nabla \cdot( & \left.\bar{A}(|x|)|\nabla u|^{\alpha(|x|)-1} \nabla u\right)-\bar{A}(|x|)(\log |u|)|\nabla u|^{\alpha(|x|)-1} \nabla \alpha(|x|) \cdot \nabla u \\
& +|\nabla u|^{\alpha(|x|)-1} \bar{A}(|x|) B_{0}(|x|) \frac{x_{i}}{|x|} \cdot \nabla u+\bar{C}(|x|)|u|^{\alpha(|x|)-1} u .
\end{aligned}
$$

We define the half-linear ordinary differential operator $q_{0}$ by

$$
\begin{gathered}
q_{0}[y]:=\quad\left(r^{n-1} \bar{A}(r)\left|y^{\prime}\right|^{\alpha(r)-1} y^{\prime}\right)^{\prime}-r^{n-1} \bar{A}(r)(\log |y|)\left|y^{\prime}\right|^{\alpha(r)-1} \alpha^{\prime}(r) y^{\prime} \\
+r^{n-1} \bar{A}(r) B_{0}(r)\left|y^{\prime}\right|^{\alpha(r)-1} y^{\prime}+r^{n-1} \bar{C}(r)|y|^{\alpha(r)-1} y,
\end{gathered}
$$

and the domain $\mathcal{D}_{q_{0}}\left(\left(r_{1}, r_{2}\right)\right)$ of $q_{0}$ is defined to be the set of all functions $y$ of class $C^{1}\left[r_{1}, r_{2}\right]$ such that $r^{n-1} \bar{A}(r)\left|y^{\prime}\right|^{\alpha(r)-1} y^{\prime} \in C^{1}\left(r_{1}, r_{2}\right) \cap C\left[r_{1}, r_{2}\right]$. If $y(r)$ is a solution of $y q_{0}[y] \geq 0$, then $u(x)=y(|x|)$ is a radially symmetric solution of $u \tilde{q}[u] \geq 0$.

Theorem 3.2. Assume that the hypotheses $\left(\mathrm{H}_{3}\right),\left(\mathrm{H}_{4}\right)$ hold. If there exists a function $z=z(r) \in \mathcal{D}_{q_{0}}\left(\left(r_{1}, r_{2}\right)\right)$ such that:

(i) $z\left(r_{1}\right)=z\left(r_{2}\right)=0$ and $z(r)>0$ in $\left(r_{1}, r_{2}\right)$;

(ii) there is a function $f_{0}=f_{0}(r) \in C\left[r_{1}, r_{2}\right]$ such that $f_{0} \in C^{1}\left(r_{1}, r_{2}\right)$ and

$$
f_{0}^{\prime}(r)=\frac{\log |z(r)|}{\alpha(r)+1} \alpha^{\prime}(r)-\frac{B_{0}(r)}{\alpha(r)+1} \quad \text { in }\left(r_{1}, r_{2}\right)
$$

(iii) $e^{f_{0}} z \in \mathcal{D}_{q_{0}}\left(\left(r_{1}, r_{2}\right)\right)$ and

$$
\left(e^{f_{0}} z\right) q_{0}\left[e^{f_{0}} z\right] \geq 0 \quad \text { in }\left(r_{1}, r_{2}\right),
$$

then every solution $v \in \mathcal{D}_{Q}(G)$ of (2.2) must vanish at some point of $\bar{G}$. 
Proof. Suppose on the contrary, that there is a solution $v \in \mathcal{D}_{Q}(G)$ of (2.2) such that $v$ has no zero on $\bar{G}$. Defining

$$
u(x):=z(|x|),
$$

we compare $u \tilde{q}[u] \geq 0$ with (2.2). The condition (ii) implies that the hypotheses $\left(\mathrm{H}_{1}\right),\left(\mathrm{H}_{2}\right)$ are satisfied for the case where $u(x)=z(|x|), f=F=f_{0}(|x|)$ and

$$
\bar{A}(|x|) B_{0}(|x|) \frac{x_{i}}{|x|} / \bar{A}(|x|)=B(x) / A(x)=B_{0}(|x|) \frac{x_{i}}{|x|} .
$$

Noting that

$$
\begin{aligned}
& \left|\nabla u+\frac{u \log |u|}{\alpha(x)+1} \nabla \alpha(x)-\frac{u}{(\alpha(x)+1) \bar{A}(|x|)} \bar{A}(|x|) B_{0}(|x|) \frac{x_{i}}{|x|}\right|^{\alpha(x)+1} \\
= & \left|\nabla u+\frac{u \log |u|}{\alpha(x)+1} \nabla \alpha(x)-\frac{u}{(\alpha(x)+1) A(x)} B(x)\right|^{\alpha(x)+1} \\
= & \left|z^{\prime}(r)+\frac{z(r) \log |z(r)|}{\alpha(r)+1} \alpha^{\prime}(r)-\frac{z(r)}{\alpha(r)+1} B_{0}(r)\right|^{\alpha(r)+1} \quad \text { on } S_{r},
\end{aligned}
$$

we easily arrive at

$$
\begin{aligned}
& V_{A\left(r_{1}, r_{2}\right)}[u] \\
& =\int_{A\left(r_{1}, r_{2}\right)}[(\bar{A}(|x|)-A(x)) \times \\
& \times\left|\nabla u+\frac{u \log |u|}{\alpha(x)+1} \nabla \alpha(x)-\frac{u}{\alpha(x)+1} B_{0}(|x|) \frac{x_{i}}{|x|}\right|^{\alpha(x)+1} \\
& \left.+(C(x)-\bar{C}(|x|))|u|^{\alpha(x)+1}\right] d x \\
& =\int_{r_{1}}^{r_{2}} \int_{S_{r}}[(\bar{A}(|x|)-A(x)) \times \\
& \times\left|\nabla u+\frac{u \log |u|}{\alpha(x)+1} \nabla \alpha(x)-\frac{u}{\alpha(x)+1} B_{0}(|x|) \frac{x_{i}}{|x|}\right|^{\alpha(x)+1} \\
& \left.+(C(x)-\bar{C}(|x|))|u|^{\alpha(x)+1}\right] d S d r \\
& =\int_{r_{1}}^{r_{2}} \int_{S_{1}}[(\bar{A}(r)-A(r, \theta)) \times \\
& \times\left|z^{\prime}(r)+\frac{z(r) \log |z(r)|}{\alpha(r)+1} \alpha^{\prime}(r)-\frac{z(r)}{\alpha(r)+1} B_{0}(r)\right|^{\alpha(r)+1} \\
& \left.+(C(r, \theta)-\bar{C}(r))|z(r)|^{\alpha(r)+1}\right] r^{n-1} d \omega d r
\end{aligned}
$$




$$
\begin{aligned}
=\omega_{n} \int_{r_{1}}^{r_{2}}\left[\left(\bar{A}(r)-\frac{1}{\omega_{n}} \int_{S_{1}} A(r, \theta) d \omega\right) \times\right. & \\
& \times\left|z^{\prime}(r)+\frac{z(r) \log |z(r)|}{\alpha(r)+1} \alpha^{\prime}(r)-\frac{z(r)}{\alpha(r)+1} B_{0}(r)\right|^{\alpha(r)+1} \\
& \left.+\left(\frac{1}{\omega_{n}} \int_{S_{1}} C(r, \theta) d \omega-\bar{C}(r)\right)|z(r)|^{\alpha(r)+1}\right] r^{n-1} d r \\
= & 0 .
\end{aligned}
$$

Therefore, all hypotheses of Theorem 3.1 are satisfied, and the conclusion follows from Theorem 3.1. The proof is complete.

\section{Specializations}

In this Section we give some specializations to the case where $\alpha(x)=\alpha>0$, and the case where $n=1, b(x)=B(x) \equiv 0$.

Theorem 4.1. Let $\alpha(x)=\alpha>0$ and $b(x) / a(x), B(x) / A(x) \in C^{1}\left(G ; \mathbb{R}^{n}\right)$. Assume that there exists a nontrivial function $u \in C^{1}(\bar{G} ; \mathbb{R})$ such that $u=0$ on $\partial G$, and that the following hypotheses are satisfied:

$\left(\hat{\mathrm{H}}_{1}\right)$ there is a function $f \in C(\bar{G} ; \mathbb{R})$ such that $f \in C^{1}(G ; \mathbb{R})$ and

$$
\nabla f=-\frac{b(x)}{(\alpha+1) a(x)} \quad \text { in } G
$$

$\left(\hat{\mathrm{H}}_{2}\right)$ there exists a function $F \in C(\bar{G} ; \mathbb{R})$ such that $F \in C^{1}(G ; \mathbb{R})$ and

$$
\nabla F=-\frac{B(x)}{(\alpha+1) A(x)} \quad \text { in } G .
$$

If $e^{f} u \in \mathcal{D}_{q}(G)$,

$$
\left(e^{f} u\right) q\left[e^{f} u\right] \geq 0 \quad \text { in } G
$$

and

$$
\begin{aligned}
V_{G}[u]=\int_{G}[ & a(x)\left|\nabla u-\frac{u}{(\alpha+1) a(x)} b(x)\right|^{\alpha+1} \\
& -A(x)\left|\nabla u-\frac{u}{(\alpha+1) A(x)} B(x)\right|^{\alpha+1} \\
& \left.+(C(x)-c(x))|u|^{\alpha+1}\right] d x \geq 0,
\end{aligned}
$$

then every solution $v \in \mathcal{D}_{Q}(G)$ of (2.2) must vanish at some point of $\bar{G}$. 
Proof. Since $\nabla \alpha(x) \equiv 0$ on $\bar{G}$, the Picone identity (2.18) holds without the hypothesis that $u$ has no zero in $G$. Therefore, the conclusion follows from Theorem 3.1.

The following corollary was established by Dunninger [12], Kusano, Jaroš and Yoshida [19].

Corollary 4.1. Let $\alpha(x)=\alpha>0$ and $b(x)=B(x) \equiv 0$ in $G$. If there exists a nontrivial function $u \in \mathcal{D}_{q}(G)$ such that $u=0$ on $\partial G, u q[u] \geq 0$ in $G$, and

$$
V_{G}[u]=\int_{G}\left[(a(x)-A(x))|\nabla u|^{\alpha+1}+(C(x)-c(x))|u|^{\alpha+1}\right] d x \geq 0,
$$

then every solution $v \in \mathcal{D}_{Q}(G)$ of (2.2) must vanish at some point of $\bar{G}$.

Proof. Since $b(x)=B(x) \equiv 0$ on $\bar{G}$, we can choose $f=F \equiv 0$ on $\bar{G}$. Hence, the conclusion follows from Theorem 4.1.

Next we consider the special case where $n=1, b(x)=B(x) \equiv 0$, that is, we let $x_{1}=t, G=\left(t_{1}, t_{2}\right)$, and define $q_{1}$ and $Q_{1}$ by

$$
\begin{aligned}
q_{1}[y]:= & \left(a(t)\left|y^{\prime}\right|^{\alpha(t)-1} y^{\prime}\right)^{\prime}-a(t)(\log |y|)\left|y^{\prime}\right|^{\alpha(t)-1} \alpha^{\prime}(t) y^{\prime} \\
+c(t)|y|^{\alpha(t)-1} y, & \\
Q_{1}[z]:= & \left(A(t)\left|z^{\prime}\right|^{\alpha(t)-1} z^{\prime}\right)^{\prime}-A(t)(\log |z|)\left|z^{\prime}\right|^{\alpha(t)-1} \alpha^{\prime}(t) z^{\prime} \\
+ & C(t)|z|^{\alpha(t)-1} z,
\end{aligned}
$$

where the coefficients appearing in (4.1) and (4.2) are supposed to satisfy the same conditions as in Section 2. The domains $\mathcal{D}_{q_{1}}(I), \mathcal{D}_{Q_{1}}(I)$ are defined as in Section 2, where $I=\left(t_{1}, t_{2}\right)$.

Theorem 4.2. Let $\alpha(x) \in C^{2}(I ;(0, \infty)) \cap C^{1}(\bar{I} ;(0, \infty))$. Assume that there exists a function $y \in C^{1}(\bar{I} ; \mathbb{R})$ such that $y\left(t_{1}\right)=y\left(t_{2}\right)=0$, y has no zero in $I$, and the following hypothesis is satisfied:

$\left(\overline{\mathrm{H}}_{1}\right)$ there is a function $f \in C(\bar{I} ; \mathbb{R})$ such that $f \in C^{1}(I ; \mathbb{R})$ and

$$
f^{\prime}(t)=\frac{\log |y|}{\alpha(t)+1} \alpha^{\prime}(t) \quad \text { in } I .
$$

If $e^{f} y \in \mathcal{D}_{q_{1}}(I)$,

$$
\left(e^{f} y\right) q_{1}\left[e^{f} y\right] \geq 0 \quad \text { in } I
$$

and

$$
\begin{gathered}
V_{I}[u]=\int_{I}[( \\
(t)-A(t))\left|y^{\prime}+\frac{y \log |y|}{\alpha(t)+1} \alpha^{\prime}(t)\right|^{\alpha(t)+1} \\
\left.+(C(t)-c(t))|y|^{\alpha(t)+1}\right] d t \geq 0
\end{gathered}
$$

then every solution $z \in \mathcal{D}_{Q_{1}}(I)$ of $z Q_{1}[z] \leq 0$ must vanish at some point of $\bar{I}$. 
Proof. The conclusion follows from Theorem 3.1.

We state the analogue of Corollary 3.1.

Corollary 4.2. Let $\alpha(x) \in C^{2}(I ;(0, \infty)) \cap C^{1}(\bar{I} ;(0, \infty))$. Assume that there is a function $y \in C^{1}(\bar{I} ; \mathbb{R})$ such that $y\left(t_{1}\right)=y\left(t_{2}\right)=0$, y has no zero in $I$, and the hypothesis $\left(\overline{\mathrm{H}}_{1}\right)$ of Theorem 4.2 holds. If $e^{f} y \in \mathcal{D}_{q_{1}}(I)$,

$$
\left(e^{f} y\right) q_{1}\left[e^{f} y\right] \geq 0 \quad \text { in } I,
$$

and

$$
a(t) \geq A(t), \quad C(t) \geq c(t) \quad \text { in } I,
$$

then every solution $z \in \mathcal{D}_{Q_{1}}(I)$ of $z Q_{1}[z] \leq 0$ must vanish at some point of $\bar{I}$.

\section{References}

[1] W. Allegretto, Sturm theorems for degenerate elliptic equations, Proc. Amer. Math. Soc. 129 (2001) 3031-3035.

[2] W. Allegretto, Form estimates for the $p(x)$-Laplacian, Proc. Amer. Math. Soc. 135 (2007) 2177-2185.

[3] W. Allegretto, Y.X. Huang, A Picone's identity for the $p$-Laplacian and applications, Nonlinear Anal. 32 (1998) 819-830.

[4] W. Allegretto, Y.X. Huang, Principal eigenvalues and Sturm comparison via Picone's identity, J. Differential Equations 156 (1999) 427-438.

[5] C. Alves, Existence of solution for a degenerate $p(x)$-Laplacian equation in $\mathbb{R}^{N}$, J. Math. Anal. Appl. 345 (2008) 731-742.

[6] G. Bognár, O. Došlý, The application of Picone-type identity for some nonlinear elliptic differential equations, Acta Math. Univ. Comenian. 72 (2003) 45-57.

[7] G. Dai, Infinitely many solutions for a $p(x)$-Laplacian equation in $\mathbb{R}^{N}$, Nonlinear Anal. 71 (2009) 1133-1139.

[8] G. Dai, Infinitely many non-negative solutions for a Dirichlet problem involving $p(x)$-Laplacian, Nonlinear Anal. 71 (2009) 5840-5849.

[9] O. Došlý, The Picone identity for a class of partial differential equations, Math. Bohem. 127 (2002) 581-589.

[10] O. Došlý, Half-linear Differential Equations, Handbook of Differential Equations: Ordinary Differential Equations, Volume 1, Elsevier B. V., Amsterdam, 2004.

[11] O. Došlý and P. Řehák, Half-linear Differential Equations, North-Holland Mathematics Studies, 202, Elsevier Science B.V., Amsterdam, 2005. 
[12] D. R. Dunninger, A Sturm comparison theorem for some degenerate quasilinear elliptic operators, Boll. Un. Mat. Ital. A (7) 9 (1995), 117-121.

[13] X. L. Fan and Q. H. Zhang, Existence of solutions for $p(x)$-Laplacian Dirichlet problem, Nonlinear Anal. 52 (2003) 1843-1852.

[14] Y. Fu and X. Zhang, A multiplicity result for $p(x)$-Laplacian problem in $\mathbb{R}^{N}$, Nonlinear Anal. 70 (2009) 2261-2269.

[15] M. Galewski, On the existence and stability of solutions for Dirichlet problem with $p(x)$-Laplacian, J. Math. Anal. Appl. 326 (2007) 352-362.

[16] M. Galewski, On a Dirichlet problem with $p(x)$-Laplacian, J. Math. Anal. Appl. 337 (2008) 281-291.

[17] G. Hardy, J. E. Littlewood and G. Pólya, Inequalities, Second Edition, Cambridge University Press, 1988.

[18] J. Jaroš, T. Kusano and N. Yoshida, Picone-type inequalities for half-linear elliptic equations and their applications, Adv. Math. Sci. Appl. 12 (2002), 709-724.

[19] T. Kusano, J. Jaroš and N. Yoshida, A Picone-type identity and Sturmian comparison and oscillation theorems for a class of half-linear partial differential equations of second order, Nonlinear Anal. 40 (2000) 381-395.

[20] M. Růžička, Electrorheological Fluids: Modeling and Mathematical Theory, Lecture Notes in Mathematics, vol. 1748, Springer-Verlag, Berlin, 2000.

[21] N. Yoshida, Oscillation criteria for half-linear partial differential equations via Picone's identity, Proceedings of Equadiff 11 (Bratislava, 2005), pp. 589-598, Bratislava, 2007.

[22] N. Yoshida, Oscillation Theory of Partial Differential Equations, World Scientific Publishing Co. Pte. Ltd., 2008.

[23] N. Yoshida, Sturmian comparison and oscillation theorems for a class of half-linear elliptic equations, Nonlinear Anal. 71 (2009) e1354-e1359.

[24] N. Yoshida, A Picone identity for half-linear elliptic equations and its applications to oscillation theory, Nonlinear Anal. 71 (2009) 4935-4951.

[25] Q. H. Zhang, Existence of radial solutions for $p(x)$-Laplacian equations in $\mathbb{R}^{N}$, J. Math. Anal. Appl. 315 (2006) 506-516.

[26] Q. H. Zhang, Oscillatory property of solutions for $p(x)$-Laplacian equations, J. Inequal. Appl. 2007 (2007), Article ID 58548, 8 pages.

[27] V. V. Zhikov, Averaging of functionals of the calculus of variations and elasticity theory, Math. USSR Izv. 29 (1987) 33-66. 